\title{
Assessment of communication words during dental treatment requiring with and without local anaesthesia between child and pediatric dentist
}

\author{
Shital Kiran Davangere Padmanabh ${ }^{1}$, (D) Para Dave ${ }^{2}$
}

\section{Highlights}

Regardless of local anaesthesia, communication played a vital role between pediatric dentist and child in the behaviour management during dental procedures.

\section{Professor \\ Dept. of Pedodontics and Preventive \\ Dentistry, College of Dental Sciences \\ At. Amargardh, Tal. Sihor, Dist Bhavnagar \\ Gujarat -364210 \\ 2 Post Graduate student \\ Dept. of Pedodontics and Preventive \\ Dentistry, College of Dental Sciences \\ At. Amargardh, Tal. Sihor, Dist Bhavnagar \\ Gujarat -364210}

\section{Correspondence:}

Dept. of Pedodontics and

Preventive Dentistry, College of

Dental Sciences, At. Amargardh, Tal.

Sihor, Dist Bhavnagar

Gujarat -364210

E-mail address:

drskiran@gmail.com

Received: 06 February 2021

Accepted: 26 April 2021

Online First: 27 April 2021
Treatment with local anaesthesia and no local anaesthesia, the words answered by the child were "na", "bmm", "burt", and "ba", "aaa", "ok" respectively.
Pediatric dentist needs to talk more often as possible in a directive to carry out successful behaviour managament regardless of the age of the child.

\section{Abstract}

Aim: To evaluate the widely used words by children and pediatric dentist during different dental procedures that involves treatment under local anaesthesia (LA) and without LA. Methods: 40 children aged between 612 years were divided in to 2 groups, Group; I treated under local anaesthesia and Group II without anaesthesia. Each group comprising of 20 subjects (male-10) (female -10) were recruited from the Department of Pediatric and Preventive Dentistry. The procedure was randomized only in one appointment by collecting the data conducted by recording the conversations between the child and dentist from the time the child walks in the dental operator until the session got over. The conversation was taped, transcribed and analyzed linguistically and statistically using chi-square test. Results: A total of 50 words were used with a minimum of 15 words in a session. There were no significant difference were found in words spoken by the child and the practitioner regarding gender, session, and duration of being acquainted with $(p>0.05)$. Regarding age groups, (4-7-year-old) significantly used fewer words than the schoolers $(6-12$-year-old) $(p<0.05)$. Conclusions: This study proved that the most commonly used words by the practitioner in treatment under LA and without LA were "syringe", "pain" and "to identify" and "stop", "open your mouth", and "to identify" respectively.

Keywords: Child; Dentist; Communication; Verbal Behavior 


\section{INTRODUCTION}

Noninvasive behaviour shaping in the form of Communication places a vital role in the behaviour management of the children. Behaviour management is defined as that procedure which very slowly develops behaviour by reinforcing successive approximations of the desired conduct until it becomes to be. ${ }^{1}$ Behaviour shaping alters conduct according to the established principles based on a learning modem. Behaviour shaping requires positive behaviour throughout the procedure and it also retraces the steps in the form of positive reinforcement. It is a fact that establishing communication will lead to the successful management of the children. The children, when they meet the new people initially, they tend to be shy and reluctant to talk, However once children are comfortable in the familiar environment, they will gain confidence. In very young children, pediatric dentist has to use euphemisms which is like a second language for most pediatric dentist. ${ }^{1}$

Behaviour management is a part of pain-free local anaesthesia with the focus of controlling the child according to the concept of perceived control which regulates the pressure of an injection discomfort. Motor signaling such as lifting a hand ${ }^{2}$ or vocal signaling such as saying "Aaaa" may be used in the perceived controlled technique. ${ }^{3}$ Use of vocal signaling that is "Aaaa" is a natural reaction when the pain is perceived. The use of this natural signal can encourage children to overcome hesitation, thus cooperate with dental treatment. Communication (i.e., opinions, or information or interchange of thoughts or imparting) may ensue by various means but, however, in dental setup, it is proficient primarily via body language, tone of voice through dialogue and facial expression. ${ }^{4}$ Perceived control is the liberty fetched to children, which permits them to have control over the dental treatment. By utilizing stop signal by either saying "aaaaa or raising hand", the pediatric dentist gives a pause in the treatment procedure. Practicing perceived control brings down children's anxiety; thereby enhancing pain-free comfortable treatment session. ${ }^{5-7}$ A child's cognitive development will dictate the level and amount of information interchange that can take place. ${ }^{8}$

Communicative management of behaviour management is the essential component of Communication that requires no specific consent, whereas all other behaviour guidance techniques necessitate informed consent. Consistent with the American Association of Pediatric Dentistry (AAPD)'s guideline on informed consent, communicative supervision and proper use of commands are pragmatic unanimously in pediatric dentistry with both the uncooperative and cooperative child. ${ }^{9}$ AAPD guidelines words highlighted the importance of 'what is told' by the practitioner and 'what is understood' by the child. Language, verbal Communication, plays a significant role in the interface between the child patient and pediatric dentist. Dental procedures may provoke anxiety and fear. Very young age children are more disposed to dental fear due to lack of coping experience. ${ }^{10}$ Behaviour management technique along with appropriate communication between the child and the clinician, ensures reduction in the child's anxiety level.

The purpose of the study was to evaluate widely used words by children and pediatric dentist during different dental procedures, which involved treatment under local anaesthesia and without anaesthesia. The null hypothesis was to test whether the treatment requiring local anaesthesia or without anaesthesia required more communication between child and pediatric dentist.

\section{METHODS}

\section{Data source}

The study protocol was approved by the local institutional ethical committee board as per the 
Helsinki declaration of human rights (Ref.No:CODS/IEC/128/2019 date 30.07.2019). Written consent was obtained from all the parents after explaining the objective of the study. This cross-sectional study adhered to Consolidated Standards of Reporting Trials (CONSORT) Guidelines and was conducted at department of pediatric and preventive dentistry, college of dental science, Amargadh, Gujarat, India, between December 2019 and January 2020.

\section{Sample size determination}

The total sample sizes were determined with an error rate of $5 \%$ with $90 \%$ power. Therefore, the minimum required samples per group were found to be 20 .

\section{Data collection}

40 children who reported to pediatric dentistry department aged between 6-12 years old were recruited by the principle investigator randomly selected the subjects using coin toss method (one patient- one appointment- one procedural session) and were divided into 2 groups namely, Group I: children were treated under local anaesthesia ( $n=20)$; Group II: children without local anaesthesia dental procedures $(n=20)$. Children aged between 6-12 years of age and their parents who gave informed consent for their child were included in the study. Speciallyabled children and children with systemic conditions were excluded.

\section{Recruitment settings}

A hidden audio recorder (Sony, Tokyo, Japan, ICD-PX240 MP3 digital voice recorder) was kept near the operator's chair to record the conversation between the pediatric dentist and the child.Subjects who reported during the first session of the clinical hours ( 9 am to $12.00 \mathrm{pm}$ ) were allocated under Group I (local anaesthesia). Similarly, subjects who reported during the second session (1.00 pm to $4.00 \mathrm{pm}$ ) were assigned under group II (without local anaesthesia).

The different treatment which was done under local anaesthesia lignocaine 2\% (ICPA health products limited, Ankleshwar, Gujarat, India) were pulp therapies, tooth extractions, a restoration, which required local anaesthesia and stainless-steel crown placement. Similarly, the various treatments which were done without local anaesthesia were oral prophylaxis, topical fluoride application, a restoration which did not require local anaesthesia and fixed space maintainers. After completion of each procedure, the words which was spoken was played on the audio speaker (JBL Flip 3 by Harman, Los Angeles, California, United States), and all the conversed words were identified in their native language and converted to English language.

Data were subjected to statistical analysis by using the chi-square test for intergroup and intragroup. A p-value $<0.05$ was considered statistically significant.

\section{RESULTS}

The pediatric dentist used a total of 10,480 words throughout the study. Children who participated in the study were aged between 6-12 years with the mean age of Group I was 9.00 years, and group II were 9.20 years. A total of 2,895 different words were spoken by the pediatric dentist, whereas the child spoke 1,980 words as a response (Table1 and Table 2).

Comparison of various words used among males and females in Group I as well as in Group II was insignificant. Comparing of various words used between the Group I and II, the chi-square value was 40 and 17.1 for the word "tooth" and "syringe", respectively. Similarly, for the "pain" the chi-square value was 29.5 and "dental cement" it was 13.9. It were observed that the word's "tooth", "pain", "syringe", and "dental cement", were found highly significant $(p=0.001)$ (Table3). 
In case of treatment done under local anaesthesia, "pain" was the most spoken word by the pediatric dentist, and the least spoken word was "this" in the entire Group I. Likewise, the response of the child to the word "pain" were noticed more, and the least responded word to the "this" was "this" among the top ten most used words by the practitioner and the child patient.

Table 1. Ten most used words by the practitioner and the child response under local anesthesia

\begin{tabular}{llll}
\hline Practitioner & $n$ & Child & $n$ \\
\hline Open your mouth & 468 & Aa & 59 \\
Stop & 290 & $\mathrm{Ha}$ & 108 \\
Keep mouth open & 550 & Aaaaa & 203 \\
Tooth & 555 & Pain & 200 \\
Syringe & 868 & Na & 500 \\
Let's check & 489 & Ok & 387 \\
Pipe & 678 & Hurts & 576 \\
Pain & 890 & Hmmm & 708 \\
This & 59 & This & 22 \\
To identify & 788 & Hmm & 678 \\
\hline
\end{tabular}

Likewise, without local anaesthesia "stop" was the most spoken word by the pediatric dentist and the least spoken word was "that" in the entire Group II. Similarly, the response of the child to the word "stop" were noticed more, and the least responded word to the "that" was "mom" among the top ten most used words by the practitioner and the child patient.

Table 2. Ten most used words by the practitioner and the child response during treatment without local anaesthesia

\begin{tabular}{llll}
\hline Practitioner & $n$ & Child & $n$ \\
\hline Open your mouth & 450 & Aa & 368 \\
To take care & 344 & Ok & 245 \\
Name & 443 & Reply & 345 \\
Cement & 398 & Hmm & 234 \\
Airotor & 449 & Na & 356 \\
To identify & 455 & Ok & 289 \\
This & 243 & Tooth & 189 \\
That & 87 & Mom & 46 \\
Pipe & 459 & Hurts & 356 \\
Stop & 589 & Ha & 467 \\
\hline
\end{tabular}

Table 3. Comparison of different variables among both groups using chi-square test

\begin{tabular}{|c|c|c|c|c|c|c|c|c|c|c|c|}
\hline \multirow[t]{2}{*}{ Group } & & \multicolumn{2}{|l|}{ Tooth } & \multicolumn{2}{|l|}{ Syringe } & \multicolumn{3}{|l|}{ Cement } & \multicolumn{2}{|l|}{$\begin{array}{l}\text { Tender on } \\
\text { percussion }\end{array}$} & \multirow[t]{2}{*}{ Total } \\
\hline & & Pain & This & $\begin{array}{l}\text { No } \\
\text { response }\end{array}$ & $\mathrm{Na}$ & $\begin{array}{l}\text { No } \\
\text { response }\end{array}$ & Hee & Hey & $\begin{array}{l}\text { No } \\
\text { response }\end{array}$ & Pain & \\
\hline \multirow[t]{2}{*}{ I } & Count & 20 & 0 & 8 & 12 & 16 & 0 & 4 & 3 & 17 & 20 \\
\hline & $\begin{array}{l}\% \text { Within } \\
\text { Group }\end{array}$ & $100 \%$ & $0 \%$ & $40 \%$ & $60 \%$ & $80 \%$ & $0 \%$ & $20 \%$ & $15 \%$ & $85 \%$ & $100 \%$ \\
\hline \multirow[t]{2}{*}{ II } & Count & 0 & 20 & 20 & 0 & 11 & 9 & 0 & 20 & 0 & 20 \\
\hline & $\begin{array}{l}\% \text { Within } \\
\text { Group }\end{array}$ & $0 \%$ & $100 \%$ & $100 \%$ & $0 \%$ & $55 \%$ & $45 \%$ & $0 \%$ & $100 \%$ & $0 \%$ & $100 \%$ \\
\hline \multirow[t]{3}{*}{ Total } & Count & 20 & 20 & 28 & 12 & 27 & 9 & 4 & 23 & 17 & 40 \\
\hline & $\begin{array}{l}\% \text { Within } \\
\text { Group }\end{array}$ & $50 \%$ & $50 \%$ & $70 \%$ & $30 \%$ & $67.5 \%$ & $22.5 \%$ & $10 \%$ & $57.5 \%$ & $42.5 \%$ & $100 \%$ \\
\hline & & $\begin{array}{l}x^{2}: 40.00 \\
p<0.001\end{array}$ & & $\begin{array}{l}x^{2}: 17.143 \\
p<0.001\end{array}$ & & $\begin{array}{c}x^{2}: 13.926 \\
p<0.001\end{array}$ & & & $\begin{array}{l}x^{2}: 29.565 \\
p<0.001\end{array}$ & & \\
\hline
\end{tabular}




\section{DISCUSSION}

When pain was anticipated, local analgesia supports behaviour management techniques (BMT)s in the dental treatment procedures. ${ }^{11}$ The regularity of linguistic techniques used throughout treatment procedure and its success in accomplishing patient cooperation have been studied. The majority of pediatric dentists agree on the importance of oral Communication to make the child's exposure to dentistry more pleasant and acceptable. Wurster et al. ${ }^{12}$ has demonstrated communication method used by the pediatric dentist depend upon behaviour of the child. Weinstein et al. ${ }^{13}$ demonstrated that inappropriate child behaviour results from ineffective approaches of the dentist, whereas the use of direction and reinforcement reduces the probability of unfavorable behaviour. They also showed the beneficial effects of empathic reactions compared to coercion and pleading.

Melamed et al. 14 tested the effect of reinforcement and concluded that it has a significant influence on the child's behaviour during dental treatment. The combination of negative and positive reinforcement consequences improved child cooperation. Dentists have used an assorted linguistic tactic during dental treatment, which has a precise outcome on the child's cooperation and behaviour. Collective strategies and different approaches would help the children overcome fear and therefore cooperating better. Verbal communication in the dental office is a part of what is termed as "institutional talk" 15 which includes communication between patient and physician which is part of a wider sphere known as "conversational analysis".16

The three models which define the features of talk between patient and physician are the activitypassivity, where the situation is controlled by the physician, especially in emergencies. Guidancecooperation, in a condition wherein the patient, permits the physician to treat, with postulation considering physician knowledge and skill required to treat, thereby the patient complies with instructions); Mutual participation, of both physician and patient make choices collectively. ${ }^{17}$ Decisions were presented, and the patient enthusiastically shares in the selecting treatment process.

Roter et al. ${ }^{18}$ advocated a supplementary model, consumerism, requests to the physician were made by the patient who is the consumer. Guidance cooperation which best designates the conversation between the child and dentist. ${ }^{19}$ Effective Communication is essential between the child and pediatric dentist to develop a trusting relationship and eventually gain the child's cooperation. ${ }^{20}$ Communication is a reciprocal process where effective Communication occurs through a must sensory approach. The dentist acts as a transmitter, and the, child acts as a receiver and spoken words as a medium. Human being learns the rules according to the verbal descriptions whereas animal acquires by environmental contingencies. Therefore, behaviour in children should be contingency control and should show scheduled effects and also Communication between practitioner and child is bipolar in nature. ${ }^{21}$

Verbal communication effectiveness in the behaviour management of the children is tremendous, along with patient satisfaction. Even any instruction advised by the paediatric dentist depends upon how the parental attitude will be towards the dentistry. The relationship between parent and child is termed as one-tailed by Bell because of the influence on developing child. Here the children behave similar to the parentalmaternal characteristic in various situation. ${ }^{22}$

By the age child reaches 5 to 6 years of age, the total vocabulary will be approximately 15,000 words with an increase of 10 words per day. According to Blinkhorn ${ }^{23}$, in 5 to 6 years old children, the total vocabulary wills 2,000 words per day. In the present study, the pediatric dentist used 2,895 different words to communicate with the child patient. In a similar study done by Caglar et al. ${ }^{24}$ in Turkish population, 626 different words 
were used for communication, which indicates that in the Indian population, pediatric dentist speaks more words for the effective communication an behaviour management of the child. Blinkhorn ${ }^{23}$ stated that the importance of greeting the child by name instead of using generalized terms for them to feel special. In the present study, the most used word by the children in group I was " $h m m m$ ", and by the pediatric dentist was "pain". Likewise, in Group II the most used word by the children was " $b a$ ", and by the pediatric dentist was "stop". This study was conducted on normal healthy children, and it would be interesting to note whether the same number of words or any variations will be observed in the case of the specially-abled children.

\section{CONCLUSIONS}

The present study proved that the most commonly used words by the practitioner in treatment under LA were "syringe", "pain", and "to identify". While the most common responded words by the child was "na", "bmm", and "burts". Similarly, the most commonly used words by the practitioner in treatment without LA were "stop", "open your mouth", and "to identify". While the most common responded words by the child patient was " $h a$ ", "Aaa", and "ok".

\section{REFERENCES}

1. Schwartz S, Kupietzky A. Local Anesthesia. In: Wright GZ, Kupietzky A editors. Behavior Management in Dentistry for Children, Second Edition. New York: John Wiley \& Sons, Inc; 2014. p.107-124

2. Chadwick BL, Honey MR. Behaviour management techniques in child training: How to Manage Children in Dental Practice. 2nd ed.London: Quintessence Publishing; 2003

3. Ram D, Kassirer J. Assessment of a palatal approach-anterior superior alveolar (P-ASA) nerve block with the Wand in Pediatric dental patients. Int J Pediatric Dent 2006:16:348-351

4. Chambers DW. Communicating with the young dental patient. J Am Dent Assoc 1976;93:793-799

5. Corah NL. Effect of perceived control on stress reduction in pedodontic patients. J Dent Res 1973;52:1261-1264

6. Weinstein P, Milgrom P, Hoskuldsson O, Golletz D, Jeffcott E, Koday M. Situation-specific child control: a visit to the dentist. Behav Res Ther 1996;34:11-21

7. Weinstein P, Raadal M, Naidu S, Yoshida T, Kvale G, Milgrom P. A videotaped intervention to enhance child control and reduce anxiety of the pain of dental injections. Eur J Paediatr Dent 2003;4:181-185

8. Goleman J. Cultural factors affecting behavior guidance and family compliance. Pediatr Dent 2014;36:121-127

9. American Dental Association Division of Legal Affairs. Dental Records [Internet] 2021 [cited 2021 Apr 10]. Available from: https://success.ada.org/en/regulatory-

legal/dental-records

10. Broberg AG, Klingberg G. Child and adolescent psychological development. In: Koch G, Poulsen S editors. Pediatric Dentistry - a clinical approach. West Sussex, UK: WileyBlackwell; 2009. p. 21-22

11. Kuscu OO, Çaglar E, Sandalli N. Local analgesia-a contemporary approach: What are the techniques that provide pain-free local analgesia for children? In: Spilieth $\mathrm{CH}$ editor. Revolutions in Pediatric Dentistry. Berlin: Quintessence Publishing; 2011. p. 135-150

12. Wurster GA, Weinstein P, Cohen AJ. Communication patterns in pedodontics. Percept Mot Skills 1979; 48:159-166

13. Weinstein P, Getz T, Ratener P, Domoto P. Dentists' responses to fear and non-fear related behaviors in children. J Am Dent Assoc 1982;104:38-40

14. Melamed BG, Bennett CG, Jerrell G, Ross SL, Bush JP, Hill C, Courts F, Ronk S. Dentists' behavior management as it affects compliance and fear in pediatric patients. J Am Dent Assoc 198;106:324-330

15. Schegloff EA. On talk and its institutional occasions. In: P Drew, J Heritage editors. Talk at 
Work. Cambridge: Cambridge University Press, 1992. p. 101-134

16. Schiffrin D. Conversation analysis. Ann Rev Appl Linguistics 1990;11:13-16

17. Szasz TS, Hollender MH. A contribution to the philosophy of medicine: The basic models of doctor-patient relationships. Arch Int Med 1956; 97:585-592

18. Roter DL, Hall JA. Doctors Talking with Patients/Patients Talking to Doctors: Improving Communication in Medical Visits.1st ed. London: Auborn House; 1992

19. Haug MR, Lavin B. Practitioner or patient Who's in charge? J Health Soc Behav 1981; 22:212-229

20. Nash DA. Engaging children's cooperation in the dental environment through effective communication. Pediatr Dent 2006; 28: 455-459

21. Broberg AG, Klingberg G. Child and adolescent psychological development. In: Koch G, Poulsen S, Pediatric Dentistry- a clinical approach. 2nd ed. West Sussex, UK: WileyBlackwell; 2009. p 21-22

22. Bell RQ. Stimulus control of parent or caretaker behavior by offspring. Dev Psychol 1971;4:63-72

23. Blinkhorn AS. Psychology of child development. In: Welbury RR. Paediatric Dentistry. 2nd ed. New York: Oxford University Press; 2001. p 23-28

24. Caglar E, Sandalli N, Kirant B, Kuscu O.O. Evaluation of words in child-paediatric dentist communication. Eur J Paediatr Dent 2015; 16: 236-238

\section{How to cite this article:}

Shital Kiran Davangere Padmanabh, and Para Dave. Assessment of communication words during dental treatment requiring with and without local anesthesia between child and pediatric dentist. Contemp Pediatr Dent 2021:2(1):14-20

\section{Declarations}

Acknowledgements: Not applicable.

Conflict of Interest Statement: The authors disclose no potential conflicts of interest.

Ethics Statement: The study protocol was approved by the local institutional ethical committee board as per the Helsinki declaration of buman rights (Ref. No:CODS/IEC/128/2019 date 30.07.2019).

Informed Consent: Informed consent and assent were obtained from all participants.

Author contributions: Conception and design: All Authors; Acquisition of data: All Authors; Interpretation of data: All Authors; Drafting article: All Author; Revision artice: SKDP; Final approval: All Authors

Funding: This work is not finantiated.

Data Availability: The data used to support the findings of this study can be made available upon request to the corresponding author.

Peer-review: Externally double-blinded peer-reviewed. 